\title{
Seismic Interferometry by cross-correlation and by multi-dimensional deconvolution using ambient seismic noise
}

\author{
Jan Thorbecke, Elmer Ruigrok, Deyan Draganov, Joost v.d. Neut, Jürg \\ Hunziker, Kees Wapenaar \\ Delft University of Technology, Department of Geotechnology, Stevinweg 1, 2628 CN Delft, The Netherlands. E-mail: \\ E.N.Ruigrok@tudelft.nl
}

Seismic interferometry (SI) is the process of retrieving new seismic responses from the crosscorrelation, cross-convolution or deconvolution of existing seismic traces. When applying SI by cross-correlation (CC), one assumes a regular source distribution. For applications in the field this will not be easily achieved. For this reason, one can make use of SI by multi-dimensional deconvolution (MDD) (Wapenaar et al., 2008). This method makes use of matrices, where a row of a matrix contains responses for a fixed receiver position and variable source positions. The Green's function is retrieved using a matrix inversion. An advantage of the MDD method is that it can compensate for irregular distribution and strength of the sources. An additional requirement of this method is that the data to be inverted need to be recorded with wide-aperture well-sampled receiver array. Contrary to this, SI by CC can be performed with recordings even at only two receivers.

When one wants to apply SI with ambient seismic noise, i.e., when no separate recordings at the receivers are possible from each of the passive seismic sources, then the matrices in the relation of SI by MDD will collapse to column vectors as each row will contain only one recording. We investigate SI by MDD with ambient noise with the help of numerical-modelling examples. We model surface waves propagating in a layered elastic medium (Wathelet et al. 2004). Next, we forward-model fundamental Rayleigh waves based on the computed dispersion curve. We use 150 simultaneously acting white-noise sources with an irregular spatial distribution, which contain two clusters of noise sources. We record the emitted noise fields at two mutually perpendicular receiver arrays. Array 1 is parallel to the source-distribution geometry and contains 129 receivers spaced at $2500 \mathrm{~m}$. Arrays 2 contains 16 receivers spaced at $20000 \mathrm{~m}$. The frequency spectrum of the noise sources peaks at $0.2 \mathrm{~Hz}$. The two arrays record the total noise for nearly 42 hours.

We choose to retrieve surface-wave information as it would be recorded along array 2 in the presence of a virtual source at the position of the middle receiver with in array 1 . We first do this using SI by CC and then use SI by MDD to retrieve the same information. MDD is a much slower process then CC. The computational time, though, can be made more acceptable if the noise traces are split into shorter noise windows. These noise windows are then arranged in columns to construct matrices, which are then used instead of the noise vectors recorded along the two receiver arrays. The MDD method is applied by least-squares inversion. SI by CC retrieves the general character of the dispersive surface waves. Nevertheless, the frequency spectrum is slightly distorted to higher frequencies and is discontinuous. These problems are consequences from the irregular source distribution. Comparing the result obtained using SI by MDD with the reference response, we see that the MDD process has corrected for these discrepancies - the spectrum is much less distorted and continuous. $\mathrm{CC}$ retrieves the kinematics of the surface wave, but the waveform is incorrectly retrieved. These artefacts result from one of the clusters in the noise-source distribution. This cluster is situated in the stationary-phase region for the virtual source in array 1 and the receivers in array 2 and causes over-illumination of that part of the region.

In conclusion, we show the application of SI by CC and by MDD to ambient seismic noise. Due to irregular distribution of the noise sources, some artefacts might arise in the CC result. SI by MDD could compensate for the irregular source distribution and suppress such artefacts. 


\section{References}

Wapenaar, K., J. van der Neut, E. Ruigrok, D. Draganov, E. Slob, and J. Thorbecke, 2008, Seismic interferometry by cross-correlation or deconvolution?: 78th Annual International Meeting, SEG, Expanded abstracts, 2731-2736.

Wathelet, M., D. Jongmans and M. Ohrnberger, 2004, Surface-wave inversion using a direct search algorithm and its application to ambient vibration measurements: Near Surface Geophysics, 2, 211221. 
